\title{
DIFFERENCES IN EARLY STOOL MICROBIOME AND THE DEVELOPMENT OF NECROTISING ENTEROCOLITIS AND SEPSIS
}

\author{
J. Berrington ${ }^{1}$, C. Stewart ${ }^{2}$, J. Perry ${ }^{3}$, N.D. Embleton ${ }^{1}$, S. Cummings ${ }^{2}$
}

${ }^{1}$ Newcastle Neonatal Service, Newcastle upon Tyne Hospitals NHS Trust, ${ }^{2}$ Biology, Food and Nutritional Sciences, University of Northumbria, ${ }^{3}$ Microbiology, Newcastle upon Tyne Hospitals NHS Trust, Newcastle upon Tyne, UK

Background: Preterm infants immune systems are educated by their gut flora, and NICU practices affect this. Available data on microbiome development in preterm infants is largely dated: newer molecular techniques offer a greater understanding of the microbiome.

Aims: To assess gut colonisation in infants $<32$ weeks; compare to existing data and explore differences in infants developing necrotising enterocolitis (NEC) or infection.

Methods: Stool (0-8 weeks) was cultured by standard culture (SC) and also had bacterial DNA extracted followed by PCR amplification (16SRNA gene) and denaturing gradient gel electrophoresis (DGGE). Resulting community profiles were analysed with diversity indices and principle component analysis.

Results: 100 (44) stools from 38 (27) infants were analysed (by SC and (DGGE)respectively). SC identified a median of 3 species/strains (range 0-8), DGGE identified a median 11 (range 3-20). 6 (1) infants were ever colonised with lactobacilli (bifidobacteria) and none with fungi (by SC). Eight infants had definite NEC (4 surgical), 7 contributed DGGE samples. Diversity index was affected by NEC (Table) and PCA separated NEC and non NEC infants.

\begin{tabular}{|l|l|l|l|}
\hline & Surgical NEC & No NEC & Medically managed NEC \\
\hline Shannon Diversity Index & 1.71 & 1.90 & 0.83 \\
\hline
\end{tabular}

[Affect on stool diversity of NEC]

Conclusions: We identified low species diversity overall, higher with DGGE than SC. Infants developing NEC had different colonisation patterns, separated by PCA. Current exposures on NICU result in a significantly abnormal microbiome and may predispose to NEC. 